IBIMA Publishing

Pediatrics Research International Journal

http://www.ibimapublishing.com/journals/PRIJ/prij.html

Vol. 2014 (2014), Article ID 955855, 4 pages

DOI: $10.5171 / 2014.955855$

Research Article

\title{
Vertebral Body Collapse at the Onset of Acute Lymphoblastic Leukemia
}

\author{
Siham Cherkaoui, Adila Hmimech, Abdellah Madani and Said Benchekroun \\ Department of Hematology and Pediatric Oncology, Ibn Rochd University hospital, Casablanca, \\ Morocco
}

Correspondence should be addressed to: Siham Cherkaoui; sihamc@gmail.com

Received Date: 10 October 2013; Accepted Date: 20 February 2014; Published Date: 27 May 2014

Academic Editor: Roberto Rivera-Luna

Copyright (C) 2014 Siham Cherkaoui, Adila Hmimech, Abdellah Madani and Said Benchekroun.

Distributed under Creative Commons CC-BY 3.0

\begin{abstract}
Bone and joint pain may be a presenting symptom in $25 \%$ of patients with acute leukemia; whereas generalised osteopenia and vertebral complications are less common.

An 11 years old boy reported a persistent low back pain since 6 weeks. The physical exam showed exquisite vertebral pain. Imaging objectified vertebral body collapse L1 and L2. Count blood cells found pancytopenia without blasts. B acute lymphoblastic leukemia was diagnosed. The boy received chemotherapy with orthopedic treatment. He was followed for two and half a year on complete remission.
\end{abstract}

Although the diagnosis of ALL was delayed because this unusual presenting complication was not recognized as such, the outcome was good.

Keywords: Acute lymphoblastic leukemia, children, vertebral collapse, back pain.

\section{Introduction}

Bone and joint pain may be a presenting symptom in $25 \%$ of patients with acute leukemia. $[1,2]$ It is recognized that children and adolescent with leukemia may initially present to the rheumatologist with leukemic arthritis. [3] Whereas generalised osteopenia and vertebral complications are less common. [1, 2]

Through this case we illustrate that acute lymphoblastic leukemia can inflict severe back pain in children without other systemic symptoms.

\section{Case}

A 11 years old boy, without past medical history, presented since 6 weeks a progressive low back pain with asthenia, without improvement under no steroid anti inflammatory drugs.

Clinical examination found a pale and afebrile child, presence of a lumbar spinal gibbus with pain, bilateral cervical lymphadenopathy $(2 \quad \mathrm{~cm})$ without hepatomegaly or splenomegaly. 
The radiography of the spine observed diffuses vertebral body collapse with cuneiform compressing of L1 (Figure 1). Spine CT scan confirmed the collapse in the anterior part of the vertebral body of L1, L2 with a slight condensation at this level without geodes, with discrete soft tissues thickening at L5 (Figure 2).
The blood count showed: the leukocyte at 2 $\mathrm{G} / \mathrm{L}$ without blasts, hemoglobin at $8.5 \mathrm{~g} / \mathrm{dl}$ and platelets at $81 \mathrm{G} / \mathrm{L}$. Bone morrow aspirate was rich with $97 \%$ of lymphoblasts. Immunophenotyping by flow cytometry confirmed the diagnosis of B-ALL.

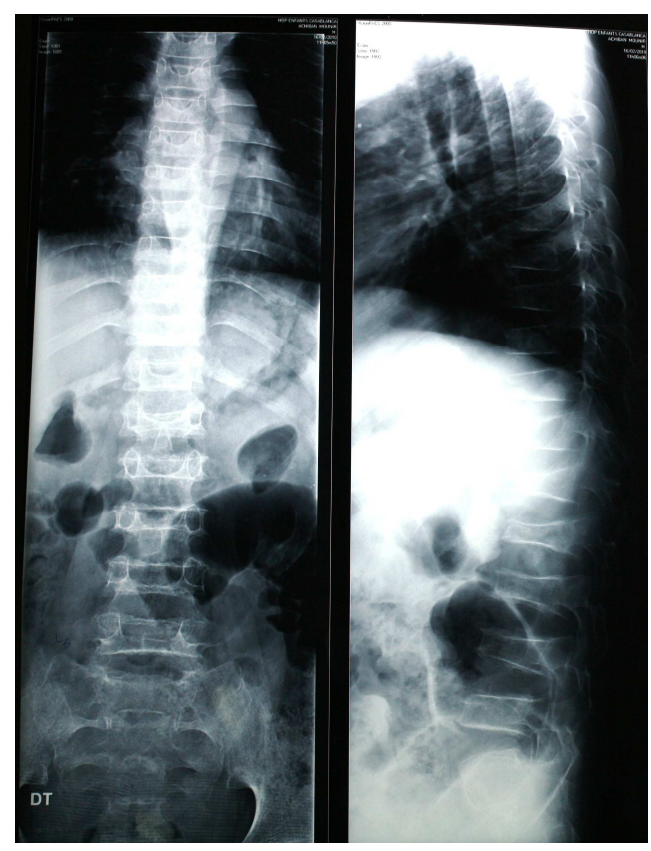

Figure 1: The Radiography of the Spine: Diffuse Vertebral Body Collapse with Cuneiform Compressing of L1.

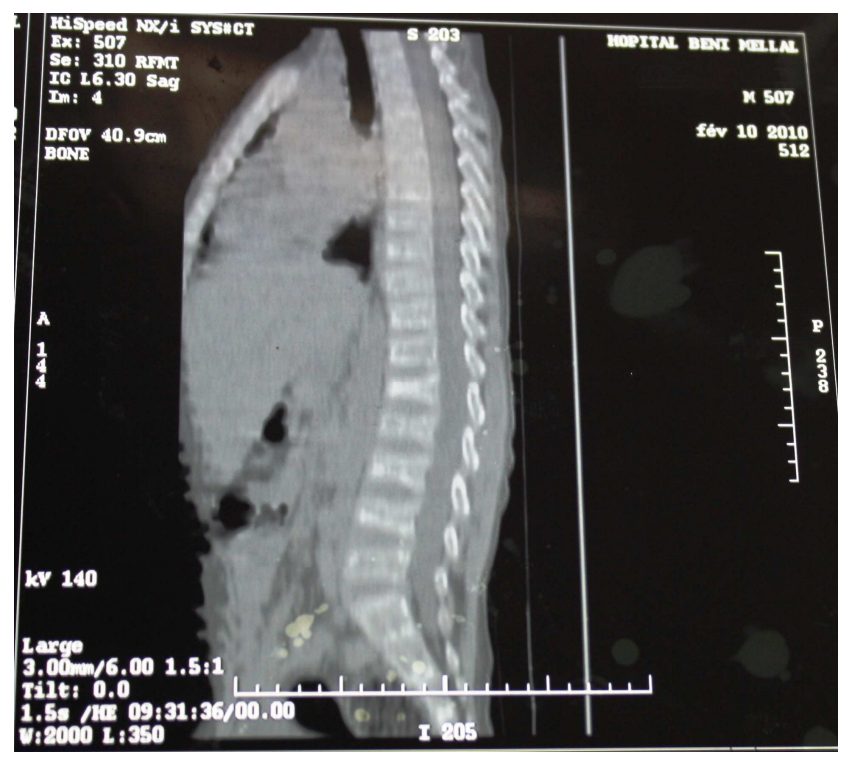

Figure 2: Spine CT Scan: Collapse in the Anterior Part of the Vertebral Body of L1, L2 with a Slight Condensation at this Level without Geodes. 
Serum calcium level was at $93 \mathrm{mg} / \mathrm{L}$ [normal: 85-105], phosphate level was at $55 \mathrm{mg} / \mathrm{L}$ [normal: 45-75], LDH level was at 365 IU / L [normal: 160-248] and spinal cerebrospinal fluid (CSF) was normal.

The child was treated with chemotherapy with a dorso lumbar Back Support. He is still in complete remission after two years and half.

\section{Discussion}

The osteoarticular manifestations are frequent in the ALL. Osteopenia, lysis of bone, lesions of osteosclerosis and periosteal reactions have been described [4-6]. Vertebral compression is rarely a first manifestation of ALL. In 1987, Samuda et al [7] reported 13 cases, and in 1996 Mandel et al [5] report 3 cases of ALL revealed by vertebral compression.

These vertebral collapses can take many radiological appearances: to be interesting all the vertebral body, the center of the vertebral body or they are cuneiform [5], as in our case.

There are a mean of 7 weeks between the discovery of the vertebral collapse and hematologic diagnosis [4]. This delay is due, firstly, to the fact that many diagnostic can discuss: isthmic spondylolysis, Schumann's disease, spondylodiscitis and bone malignancies $[5,6]$, David et al reported a retrospective study of 29 cases of children with malignancies initially addressed in pediatric rheumatology unit (juvenile chronic arthritis, spondyloarthropathies ...) [3], on the other hand, the possible absence of biological orientation with an initial normal blood count [6].

The skeletal manifestations with hypercalcemia were secondary to massive infiltration in medullary and periosteal spaces by blasts, responsible of lytic lesions by local destruction or diffuse hyper resorption, secondary to the production of a cytokine involeved in osteoclastic activation or synthesis by blasts of a parathyroid hormone-like $[4,5,8]$. The presence of the initial bone involvement in
ALL does not seem to affect the prognosis [1].

The treatment of vertebral collapse is double: orthopedic by wearing a corset for some authors [7] and the specific treatment of ALL [5, 6]. Our patient received an orthopedic treatment with chemotherapy. Complete remission is necessary for the healing of bone lesions [1].

\section{Conclusion}

This case shows that delay in diagnosis occurs often when the classic clinical manifestations of acute leukemia are absent. For such patients referred, to the pediatrician, we believe that the diagnosis of malignancy should be considered earlier.The discrete anemia and leukopenia may be the signs of the orientation in the absence of circulating blasts and must perform a bone morrow aspirate.

\section{Consent}

Patient's parents have given their informed consent for the case report to be published.

\section{References}

1- Pandya, N. A., Meller, S. T., MacVicar, D. et al. (2001). "Vertebral Compression Fractures in Acute Lymphoblastic Leukaemia and Remodeling after Treatment," Archives of Disease in Children, 85 (6) 492-493.

2- Santangelo, J. R. \& Thomson, J. D. (1999). "Childhood Leukemia Presenting with Back Pain and Vertebral Compression Fractures," The American Journal of Orthopedics, 28(4) 257-260.

3- Cabral, D. A. \& Tucker, L. B. (1999). "Malignancies in Children Who Initially Present with Rheumatic Complaints," Journal of Pediatrics, 134 (1) 53-57.

4- Roux, S. \& Mariette, X. (2005). "Bone and Joints in Haematological Malignancies (Myeloma Excluded)," EMC-Rhumatologie Orthopédie, 2 (2) 109-124 
5- Mandel, R., Vic, P., Nelker, B. et al. (1996). "Tassements Vertébraux Révélateurs de Leucémie Aigue Lymphoblastique," Archives de Pediatrie, 3 (5) 466-469.

6- Hbibi, M., Atmani, S., Abourazzak, S. et al. (2011). "Tassement Vertébral Révélant une Leucémie Aigue Chez un Enfant," Archives de Pédiatrie, 18 (5) 597-599.

7- Samuda, G. M., Cheng, M. Y. \& Yeung, C. Y. (1987). "Back Pain and Vertebral Compression: an Uncommon Presentation of Childhood Acute Lymphoblastic Leukemia," Journal of Pediatric Orthoedics, 7 (2) 175-178.

8- Davies, J. H., Evans, B. A. J., Jenney, M. E. M. \& Gregory, J. W. (2005). "Skeletal Morbidity in Childhood Acute Lymphoblastic Leukaemia," Clinical Endocrinology, 63 (1) 1-9. 